\title{
Mitigating feather pecking behavior in laying poultry production through tryptophan supplementation
}

\author{
Truong Van Hieu ${ }^{(i D}$ | Nguyen Hoang Qui ${ }^{*}$ (D) | Nguyen Thi Kim Quyen
}

aTra Vinh University, No. 126 Nguyen Thien Thanh Street, Ward 5, Tra Vinh City, Tra Vinh Province, Vietnam.

*Corresponding author: nguyenhoanquitv@gmail.com

\begin{abstract}
Feather pecking behavior is regarded as the most serious welfare concern in poultry layer production. The cause of feather pecking is partly due to the nutrient-deficient diets that further depletes the tryptophan levels in the animal's system. Tryptophan is an essential amino acid and thus, cannot be synthesized in the body of animals, including poultry. This amino acid can be metabolized through three pathways. Tryptophan metabolism produces important metabolites: serotonin and melatonin. Tryptophan affects hormone secretion in poultry. Thus, it affects poultry's psychological status, which eventually leads to feathers' pecking. The application of tryptophan as a feed additive can improve poultry performance and alleviate feather pecking behavior or stress response in poultry production. This is achieved through indole pathways and mostly through the Tryptophan-Kynurenine pathway. This review paper aims to provide detailed information regarding the performance of tryptophan on feather pecking behavior, particularly in laying poultry animals.
\end{abstract}

Keywords: animal welfare, pecking, stress, tryptophan's pathway

\section{Introduction}

The laying poultry industry has been strengthening and steadily developing recently and has been providing a large proportion of meat and eggs for human consumption. The poultry performance, specifically in egg production, is mainly affected by the ingredients used and the nutritional specifications of the diet. According to Blokhuis et al (2007), laying poultry faces many severe problems. One of them is feather pecking in the laying phase, which is a repetitive oral behavior when birds peck at the feather of other birds repetitively. The study of Riedstra and Groothuis (2004) asserted that severe feather pecking is interspersed with episodes of soft feather pecking. Among these different types of feather pecking behavior, there might be a popular core motive and/or neurobiological basis for these behaviors. One of the proposed reasons for feather pecking is a nutritional factor, specifically with a low tryptophan diet, related to the serotonergic system in developing feather pecking behavior (Savory et al 1999). Besides, it has been proposed that the precursor tryptophan and the avian serotonergic system are intricately connected with repeated feather pecking (De Haas and Eijk 2018). However, the neurobiological mechanisms behind feather pecking remain unclear. For example, featherdestructive pecking behavior in birds selected for egg production is mediated by the tryptophan (Birkl et al 2019a). In the same statement, Linh et al (2021) debated that when it comes to regulating the behavior of poultry, tryptophan is known to affect greatly, especially in stressful conditions or in a crowded environment. The chemical structure of tryptophan was provided in Figure 1.

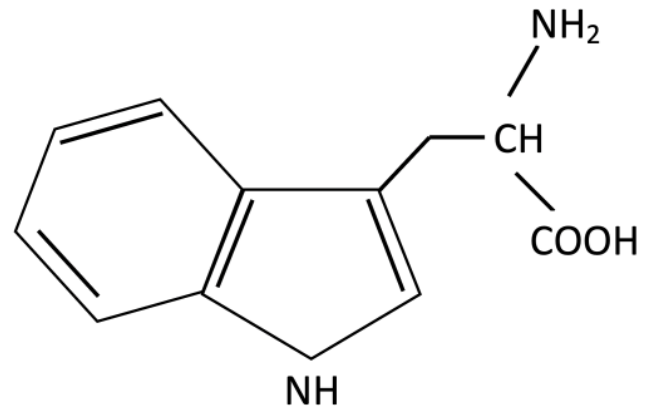

Figure 1 Tryptophan chemical structure. Source: Pubchem 6305 (2021)

Additionally, the role of tryptophan in reducing stress responses and aggressive behavior in animals was recorded in the study of Koopmans et al (2012). More clearly, the role of tryptophan in reducing feather pecking behavior in poultry animals was also shown in the studies of Birkl et al (2019a), Birkl et al (2019b), Khattak and Helmbrecht (2019) and Yıldırım et al (2020). Due to tryptophan's physiological roles, the non-inclusion of tryptophan in the diet could lead to many problems such as feather pecking behavior. Therefore, improving poultry production while reducing feather pecking under stress conditions is one of the crucial missions of scientists nowadays, and to do so, one must have a better understanding of the feather pecking behavior. It is significant to track the metabolism of amino acids, especially tryptophan in poultry, and how tryptophan affects the process of feather pecking behavior in the production of 
layers. Thus, this study aims to review the role of tryptophan in the feather pecking behavior of laying poultry and give valuable information available to the farmers.

\section{Mode of action}

In 1901, tryptophan was firstly reported by Frederick Hopkins, who discovered tryptophan through casein hydrolysis. Tryptophan can be found in some grains as its natural sources, such as walnuts, cashews, peanuts, sesame, almonds, sunflower seeds, pumpkin and soybeans with high concentrations (Strasser et al 2016). The report of Friedman (2018) also showed that high-tryptophan-transgenic seeds have a higher amount of tryptophan than normal seeds with a lower price. As widely known, tryptophan production follows three metabolic pathways (Figure 2), which are: (1) protein synthesis pathway; (2) serotonin and melatonin pathway; (3) kynurenine pathway. As with other amino acids, tryptophan is involved in the production of protein in the bodies of animals via the liver. The kynurenine route is the most significant of the three tryptophan-metabolism pathways. This synthesizes the indole molecule, which is essential in forming metabolites that have physiological activity and are associated with the body's defense (Nathalie and Seve 2007).

The body uses just about $1 \%$ to $2 \%$ of ingested tryptophan to produce serotonin under normal physiological conditions. The kynurenine pathway is the primary metabolic pathway, through which $95 \%$ of dietary tryptophan is processed and excreted (Davis and Liu 2015). By induction of tryptophan pyrrolase in the liver, most of the total ingested tryptophan is catabolized into kynurenine (Stone and Darlington 2002). Furthermore, the kynurenine pathway is the process that mainly affects feather pecking behavior. Following figure 2, if the pyrrolase is induced in the liver by the enzymes of tryptophan 2,3-dioxygenase (TDO) and indolamine 2,3-dioxygenase (IDO), the availability of tryptophan could decrease. The TDO and IDO activities could also affect serotonin production as well.

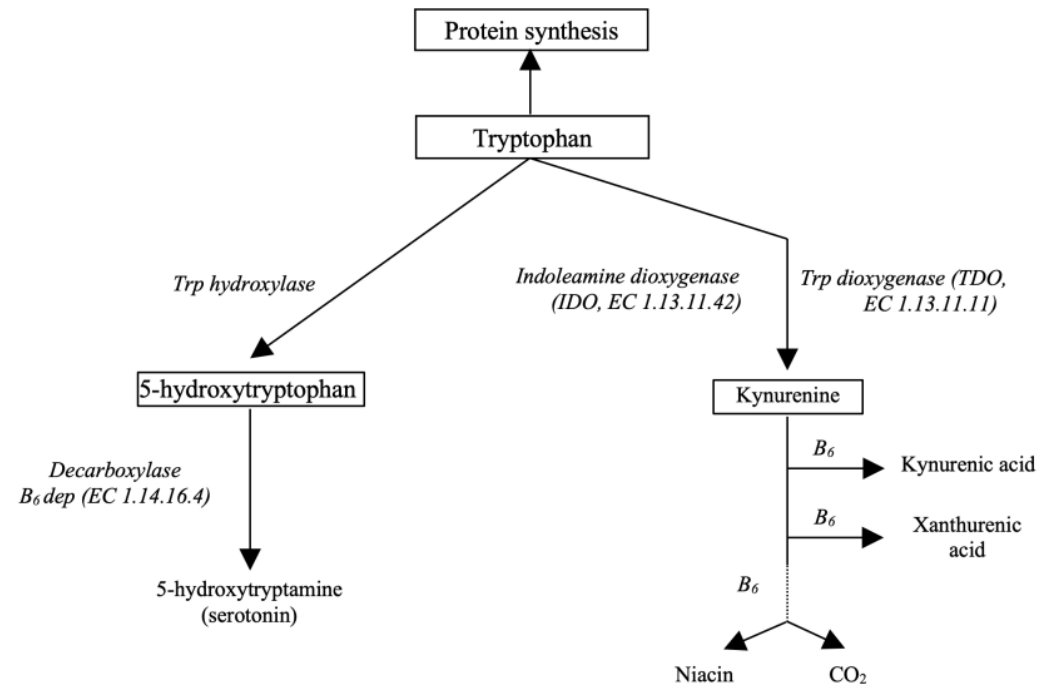

Figure 2 Tryptophan metabolic pathways. Source: Nathalie and Seve (2007).

Additionally, it has been suggested that stressful situations and immunological challenges increased the action of indolamine 2,3-dioxygenase. This results to the metabolization of tryptophan to kynurenine, thereby depleting the tryptophan in the body system, required to create serotonin in the mammalian brain (Konsman et al 2002). Since glucocorticoids and proinflammatory cytokines trigger indolamine 2,3-dioxygenase, stress is expected to impact tryptophan metabolism. Thus, it suggests that the kynurenine pathway is involved (Miura et al 2008). As widely known, tryptophan (L-Trp) is a precursor for synthesizing serotonin. It has been discovered that hens from a high feather pecking behavioral breed have a high serotonin turnover level in response to severe behavioral stress, which is lower than the poultry of a low feather pecking behavioral breed when exposed to the same stressor (Hierden et al 2002).
According to Hierden et al (2004), the dietary inclusion of tryptophan enhances serotonergic neurotransmission, leading to an increase in the tryptophan turnover to serotonin in the brain. Moreover, low serotonergic neurotransmission appears to be a possible reason for feather pecking behavior. Consequently, the boosting of the serotonergic tone by adding higher levels of tryptophan in the basal diet reduces feather pecking activity.

\section{Tryptophan in feather pecking behavior}

According to Savory (1995), feather pecking has five distinguished kinds, namely: (1) aggressive pecking, (2) severe feather pecking, (3) gentle feather pecking, (4) vent pecking, and (5) tissue pecking. Several factors affect feather pecking behavior in poultry, including internal and external factors (Figure 3 ). These factors sometimes interact with others to affect feather pecking behavior, or sometimes it acts alone in poultry production. Feeding is emphasized in 
this paper to understand the role of tryptophan in feather pecking behavior clearly. Following figure 3, external factors such as diet composition and feed strategy are two factors related to feeding. This includes essential amino acids, and tryptophan is no exception because of its importance for poultry behavior. In addition to that, tryptophan-deficient diets may negatively affect birds' feather pecking behavior (Kjaer et al 2013). This behavior is most frequently correlated with central serotonin deficiency during the laying phases. Serotonin is produced in the tryptophan metabolic pathway (Kops et al 2017; De Haas and Eijk 2018). The birds attempt to peck their feathers to overcome an amino acid deficiency in their diet. This is because the feathers contain certain important amino acids. Feather pecking in poultry is reduced when tryptophan is supplemented with a sufficient level in the diet (Savory 1999; Hierden et al 2004).

Laying poultry supplemented with a suitable amount of tryptophan improves production performance and controls feather pecking behavior. The requirement of tryptophan for layer diets following NRC guidelines is 1.6 $\mathrm{g} / \mathrm{kg}$. This is equivalent to $160 \mathrm{mg}$ tryptophan per hen based on daily feed intake (NRC 1994). Notably, supplying laying hens with a greater Trp: Lys ratio resulted in lower feather pecking behavior (Mens et al 2020).

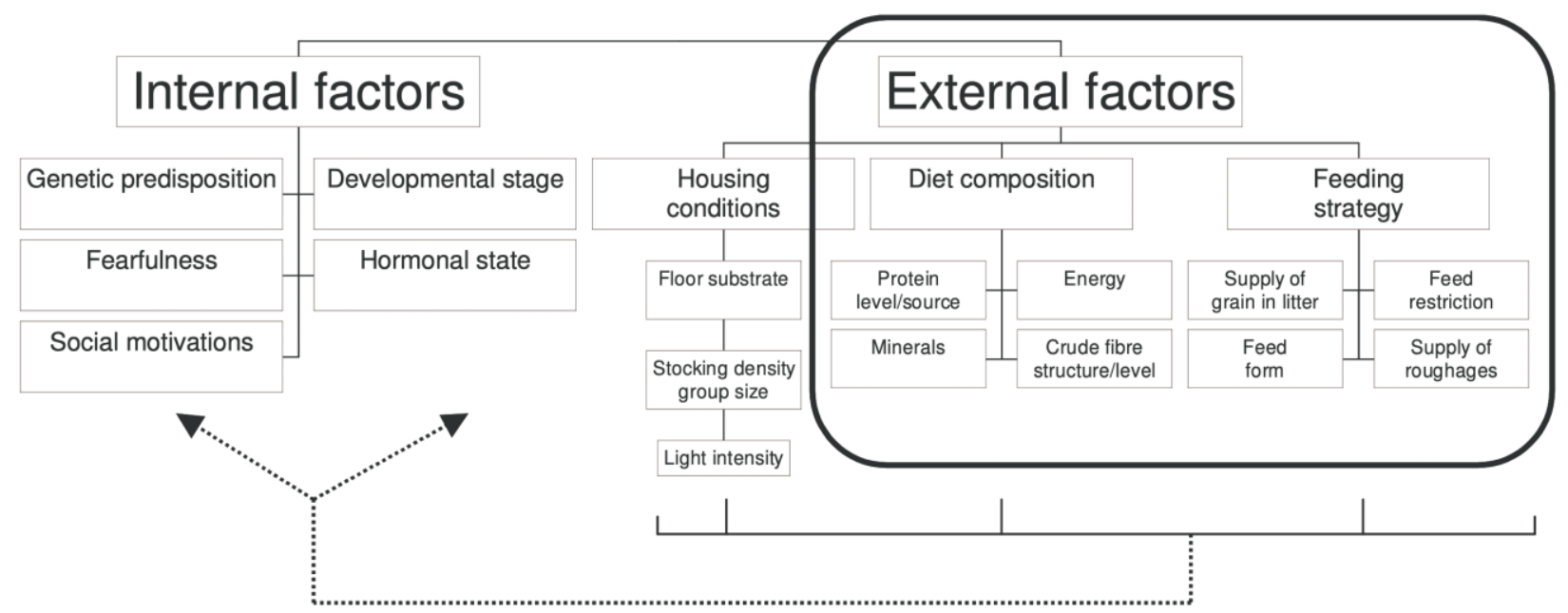

Figure 3 Overall factors affecting feather pecking behavior in poultry. Source: Van Krimpen et al (2005).

Birkl et al (2019a) demonstrated that acute tryptophan depletion in the diet enhances feather pecking in both high and low feather pecking lines, implying that dietary inclusion of tryptophan has an impact on hens' social behavior, including feather pecking. Assuming that tryptophan can directly affect central serotonin synthesis, the supplementation of tryptophan during rearing can be an effective approach of preventing feather pecking, as central serotonin levels in the brain are low during rearing (Kops et al 2017). An investigation on the tryptophan availability in plasma found out that low levels in young laying hens at 24 weeks of age were preceded by the onset of harmful and aggressive pecking (Birkl et al 2017). It appears that lower tryptophan availability in birds at week 24 may be due to increased stress levels in pens where injurious aggressive pecking started early on, according to the data of Birkl et al (2017). Acute tryptophan depletion can be thought of as an exciting non-invasive nutritional tool for investigating serotonergic involvement in various abnormal behaviors for which serotonergic (5-HT) involvement is assumed, such as repetitive feather pecking in birds. (Grindlinger and Ramsay 1991).

\section{Final considerations}

Tryptophan has a crucial role in the feather pecking behavior in all kinds of poultry species, especially during the laying period. Tryptophan and its metabolites play an indispensable role in regulating feather pecking behavior. Through the kynurenine pathway, tryptophan reduces repetitive feather pecking by a significant amount. Since tryptophan could not be synthesized enough in a mammal's body, supplementation tryptophan in the diet is an excellent choice to reduce feather pecking behavior during the laying phase. However, the information regarding the specific amount of tryptophan that can reduce feather pecking behavior is still limited. It is recommended to do further research on the role of tryptophan, specifically in poultry animals.

\section{Acknowledgements}

We would like to appreciate Tra Vinh University for supporting us in this review. We are also thankful to Mr. Mark Anthony Mercado from the Philippines for checking grammar for this paper.

\section{Conflict of Interest}

The authors have no conflicts of interest related to the present review.

\section{Funding}

This research did not receive any financial support. 


\section{References}

BirkI P, Chow J, Forsythe P, Gostner JM, Kjaer JB, Kunze WA, McBride P, Fuchs D, Harlander-Matauschek A (2019a) The Role of Tryptophan-Kynurenine in Feather Pecking in Domestic Chicken Lines. Frontiers in veterinary science 6:209.

Birkl P, Chow J, McBride P, Kjaer JB, Kunze W, Forsythe P, HarlanderMatauschek A (2019b) Effects of acute tryptophan depletion on repetitive behavior in laying hens. Frontiers in veterinary science 6:230.

Birkl P, Franke L, Bas Rodenburg T, Ellen E, Harlander-Matauschek A (2017) A Role for Plasma Aromatic Amino Acids in Injurious Pecking Behavior in Laying Hens. Physiology \& Behavior 175:88-96.

Blokhuis HJ, Van Niekerk TF, Bessei W, Elson A, Guémené D, Kjaer JB, Maria Levrino GA, Nicol CJ, Tauson R, Weeks CA, Van De Weerd HA (2007) The LayWel project: welfare implications of changes in production systems for laying hens. World's Poultry Science Journal 63:101-14

Davis I, Liu A (2015) What is the tryptophan kynurenine pathway and why is it important to neurotherapeutics? Expert Review of Neurotherapeutics 15:719-721.

Friedman M (2018) Analysis, nutrition, and health benefits of tryptophan International Journal of Tryptophan Research 11:1178646918802282.

Grindlinger HM, Ramsay E (1991) Compulsive feather picking in birds. Archives Of General Psychiatry 48:857-857.

Haas EN and Eijk JAJ (2018) Where in the serotonergic system does it go wrong? Unravelling the route by which the serotonergic system affects feather pecking in chickens. Neuroscience \& Biobehavioral Reviews 95:170188

Hierden YM Van, Korte SM, Koolhaas JM (2004) Chronic increase of dietary L- Tryptophan decreases gentle feather pecking behaviour. Applied Animal Behaviour Science 89:71-84.

Hierden YM Van, Korte SM, Ruesink EW, Reenen CGV, Engel B, Korte Bouws GAH, Koolhaas JM, Blokhuis HJ (2002) Adrenocortical reactivity and central serotonin and dopamine turnover in young chicks from a high and low feather-pecking line of laying hens. Physiology and Behavior 75:653-659.

Khattak FM, Helmbrecht A (2019) Effect of different levels of tryptophan on productive performance, egg quality, blood biochemistry, and caecal microbiota of hens housed in enriched colony cages under commercial stocking density. Poultry Science 98:2094-2104.

Kjaer JB, Bessei W (2013) The interrelationships of nutrition and feather pecking in the domestic fowl a review. European Poultry Science 77:1-9.

Konsman JP, Parnet P, Dantzer R (2002) Cytokine-induced sickness behavior: mechanisms and implications. Trends in Neuroscience 25:154-159.

Koopmans SJ, van der Staay FJ, Le Floc'h N, Dekker R, van Diepen JTM, Jansman AJM (2012) Effects of surplus dietary I-tryptophan on stress, immunology, behavior, and nitrogen retention in endotoxemic pigs. Journal of Animal Science 90:241-251.
Kops MS, Kjaer JB, Gunturkun O, Westphal KGC, Korte-Bouws GAH, Olivier B, Korte SM, Bolhuis JE (2017) Brain monoamine levels and behavior of young and adult chickens genetically selected on feather pecking. Behavioural Brain Research 327:11-20.

Linh NT, Guntoro B, Qui NH (2021) Immunomodulatory, behavioral, and nutritional response of tryptophan application on poultry. Veterinary World 14:2244-2250.

Miura H, Ozaki N, Sawada M, Isobe K, Ohta T, Nagatsu T (2008) A link between stress and depression: shifts in the balance between kynurenine and serotonin pathways of tryptophan metabolism and the etiology and pathophysiology of depression. Stress 11:198-209.

Nathalie LF, Seve B (2007) Biological roles of tryptophan and its metabolism: Potential implications for pig feeding. Livestock Science 112:23-32.

National Center for Biotechnology Information (2021) PubChem Compound Summary for CID 6305, Tryptophan. https://pubchem.ncbi.nlm.nih.gov/compound/Tryptophan. Accessed on: December 1, 2021

NRC (1994) National Research Council, Nutrient Requirements of Poultry: Ninth Revised Edition, Natl. Acad. Press, Washington, DC

Riedstra B, Groothuis TGG (2004) Prenatal light exposure affects early feather-pecking behavior in the domestic chick. Animal Behaviour 67:10371042.

Savory CJ (1995) Feather Pecking and Cannibalism. World's Poultry Science Journal 51:215-219.

Savory CJ, Mann JS, MacLeod MG (1999) Incidence of pecking damage in growing bantams in relation to food from, groups size, stocking density, dietary tryptophan concentration and dietary protein source. British Poultry Science 40:579-584

Stone TW, Darlington LG (2002) Endogenous kynurenines as targets for drug discovery and development. Nature Reviews Drug Discovery 1:609-620.

Strasser B, Gostner JM, Fuchs D (2016) Mood, food, and cognition: Role of tryptophan and serotonin Current Opinion in Clinical Nutrition and Metabolic Care 19:55-61.

Van Krimpen MM, Kwakkel RP, Reuvekamp BFJ, Van Der Peet-Schwering CMC, Den Hartog LA, Verstegen MWA (2005) Impact of feeding management on feather pecking in laying hens. World's Poultry Science Journal 61:663686.

Yıldırım A, Kraimi N, Constantin P, Mercerand F, Leterrier C (2020) Effects of tryptophan and probiotic supplementation on growth and behavior in quail. Poultry Science 99:5206-5213. 